tere Informationen beschafft, Daten erhoben und Untersuchungen durchgeführt werden, ist die Funktionsgrenze des FFH-Screenings überschritten und eine FFH-VP obligatorisch. Die Berufung auf „Irrelevanzschwellen“ kann daher nicht von der Verpflichtung entbinden, eine sorgfältige und die besten wissenschaftlichen Erkenntnisse reflektierende Verträglichkeitsprüfung durchzuführen.

2.3 Erschwerend kommt hinzu, dass selbst bei geringfügigen projektbedingten Zusatzbelastungen im Umfang von weniger als 3\% des CL-Wertes stets damit zu rechnen ist, dass derartige Einträge im Zusammenwirken mit anderen Plänen oder Projekten die „Irrelevanzschwelle“ überschreiten. $\$ 34$ Abs. 1 BNatSchG trägt diesem Umstand in voller Übereinstimmung mit der unionsrechtlichen Vorgabe des Art. 6 Abs. 3 S. 1 FFH-RL Rechnung und lässt keinen Zweifel daran, dass Projekte auch dann einer Verträglichkeitsprüfung $\mathrm{zu}$ unterziehen sind, wenn sie zwar nicht aus sich heraus, wohl aber im Zusammenwirken mit anderen Vorhaben planerischer oder projektbezogener Art geeignet sind, ein Gebiet des Netzes Natura 2000 erheblich zu beeinträchtigen. Sind daher im Rahmen einer FFHVorprüfung stets auch kumulative Effekte in den Blick zu nehmen, kann keine Rede davon sein, dass eine FFH-VP allein deshalb entbehrlich wäre, weil ein Projekt bei isolierter Betrachtung nur geringfügige Stickstoffzusatzbelastungen verursacht.

2.4 Schließlich bergen „Abschneidewerte“ der genannten Art die erhebliche Gefahr in sich, dass nährstoffsensible Lebensraumtypen in einer längerfristigen Perspektive beträchtlichen zusätzlichen Stickstoffeinträgen ausgesetzt werden, ohne dass sich die hierfür verantwortlichen Projekte einer Verträglichkeitsprüfung haben stellen müssen. Das ist namentlich dann der Fall, wenn zu irrelevanten Stickstoffbelastungen führende Projekte nicht zeitgleich, sondern in gewissen zeitlichen Abständen zugelassen werden. Gelangt die „Irrelevanzschwelle“ bei jedem Projekt zur Anwendung, wird bei keinem dieser Vorhaben eine Verträglichkeitsprüfung durchgeführt, obwohl sie im Zusammenwirken Stickstoffbelastungen hervorrufen, die jenseits der 3\%-Schwelle liegen. Dem sich damit verbindenden Problem einer zum Unterlaufen der FFH-VP führenden nachträglichen $\mathrm{Ku}-$ mulation ließe sich begegnen, wenn diese Bagatellschwelle in Ansehung eines betroffenen Gebietes stets nur einmal in
Anspruch genommen werden dürfte. Praktisch ist dies freilich ausgeschlossen, weil nirgends dokumentiert wird, bei welchen Projekten bereits zuvor auf geringfügige Zusatzbelastungen und eine Entbehrlichkeit der FFH-VP erkannt wurde. Auch aus Gründen der in anderen Zusammenhängen gelegentlich bemühten „praktischen Vernunft"13 ist es daher geboten, die Pflicht zur Verträglichkeitsprüfung nicht einem Bagatellvorbehalt zu unterstellen.

3. Schon die in der bisherigen Judikatur des Bundesverwaltungsgerichts akzeptierten „Bagatell- bzw. Irrelevanzschwellen" sehen sich Bedenken ausgesetzt, weil sie einer schleichenden Vernichtung geschützter Lebensraumtypen Vorschub leisten. ${ }^{14}$ Einzuräumen ist freilich, dass die Funktion des Bagatellvorbehalts bislang nur darin bestand, die Reichweite des durch $\$ 34$ Abs. 2 BNatSchG begründeten Verbots in Fällen zu begrenzen, in denen sich nach Durchführung einer ordnungsgemäßen FFH-VP herausstellte, dass ein Projekt die Stickstoffbelastung eines Lebensraumtyps in einem Umfang erhöht, der aus fachwissenschaftlicher Sicht zumindest keine signifikanten Veränderungen des Ist-Zustandes auslöst und die Wiederherstellung eines günstigen Zustandes nicht in signifikanter Weise einschränkt. ${ }^{15}$ Während „Irrelevanzschwellen“ dem 9. Senat des Bundesverwaltungsgerichts dazu dienen, von ihm als „überschießend“ begriffene Tendenzen der Verbotsvorschrift des $\$ 34$ Abs. 2 BNatSchG zurückzuschneiden, erinnert das Urteil des 7. Senats an den sprichwörtlichen „Elefanten im Porzellanladen", weil der Bagatellvorbehalt hier als Instrument fungiert, um schon die dem Verbot vorgelagerte Pflicht zur sorgfältigen Prüfung der nachteiligen Auswirkungen eines Projekts zu relativieren. Der Instrumentenkasten des Habitatschutzrechts wird damit ,kurzerhand vom Tisch gewischt", ohne dass dies in $\$ 34$ Abs. 1 BNatSchG einen Rückhalt fände.

13) BVerwG, Urt. v. 12.3.2008 - 9 A 3.06, NuR 2008, 633 Rdnr. 124.

14) Gellermann, Besonders geschützte Teile von Natur und Landschaft im Anlagenzulassungsverfahren, in: Thomé-Kozmiensky/Dombert/Versteyl/Rotard/Appel (Hrsg.), Immissionsschutz, Band 2, 2011, S. 165, 173.

15) BVerwG, Urt. v. 14.4.2010 - 9 A 5.08, NVwZ 2010, 1225 Rdnr. 94.

\title{
BUCHBESPRECHUNGEN
}

DOI: 10.1007/s10357-012-2219-y

\section{Der Artenschutz im Bebauungsplanverfahren}

Matthias Blessing/Eckart Scharmer: Der Artenschutz im Bebauungsplanverfahren, Verlag W. Kohlhammer 2012, 158 S., 49,90 Euro. ISBN 978-3-17-021846-8.

Dieses kleine Handbuch befasst sich mit einem Teilbereich des $\mathrm{Na}$ turschutzrechts, der für die Praxis von großer Bedeutung ist. Die Autoren stellen zunächst den Artenschutz und seine Bedeutung in der Bebauungsplanung dar. Sie behandeln dann die artenschutzrechtlichen Verbote des $\$ 44$ BNatSchG und stellen alle wichtigen Einzel-

RA Peter Fischer-Hüftle,

Regensburg, Deutschland heiten dar. Anschließend erläutern sie die Behandlung artenschutzrechtlicher Konflikte im Bebauungsplanverfahren unter besonderer Beachtung der Regelungen der $\iint 44$ Abs. 5, 45 Abs. 7 Satz 1 Nr. 5 und Satz 2 sowie 67 Abs. 2 BNatSchG. Es schließt sich ein Kapitel über die Umsetzung artenschutzrechtlicher Maßnahmen im Bebauungsplan an. Den Schlussteil bildete die Erörterung der Frage, wie der Artenschutz im Bebauungsplanverfahren zu berücksichtigen ist. Es ist den Autoren gelungen, die vielfältigen Detailfragen in ein übersichtliches Gliederungskonzept einzufügen und sehr klar und verständlich darzustellen. Dabei berücksichtigen sie die inzwischen umfangreiche Rechtsprechung insbesondere der Obergerichte. Wenn die Autoren die Rechtsprechung nicht für überzeugend halten, begründen sie das mit beachtlichen Argumenten. Für offene Fragen werden abgewogene Lösungsvorschläge angeboten. Insgesamt ein Buch, dessen Lektüre jedem, der in der Praxis mit Artenschutz und Bebauungsplanung befasst ist, Gewinn bringt und zur Anschaffung empfohlen werden kann. 